\title{
Pattern search methods framework for beam angle optimization in radiotherapy design
}

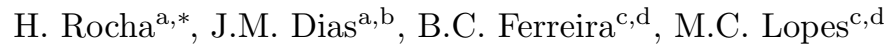 \\ ${ }^{a}$ INESCC, Rua Antero de Quental 199, 3000-033 Coimbra, Portugal. \\ ${ }^{b}$ FEUC, Av. Dias da Silva 165, 3004-512 Coimbra, Portugal. \\ ${ }^{c}$ IPOC-FG, EPE, Av. Bissaya Barreto 98, 3000-075 Coimbra, Portugal. \\ ${ }^{d}$ I3N, Campus Universitário de Santiago, 3810-193 Aveiro, Portugal.
}

\begin{abstract}
The selection of appropriate beam directions is decisive for the quality of the treatment, both for maximizing tumor doses and for organs sparing. However, the beam angle optimization (BAO) problem is still an open problem and, most of the time, beam directions continue to be manually selected in clinical practice which requires many trial and error iterations between selecting beam angles and computing fluence patterns until a suitable treatment is achieved. The goal of $\mathrm{BAO}$ is to improve the quality of the directions used and, at the same time, release the treatment planner for other tasks. The objective of this paper is to introduce a new approach for the resolution of the BAO problem, using pattern search methods to tackle this highly non-convex optimization problem. Pattern search methods are derivative-free optimization methods with the ability to avoid local entrapment. Moreover, they require few function value evaluations to progress and converge. These two characteristics gathered together make pattern search methods suited to address the BAO problem. A set of clinical examples of head-and-neck cases is used to discuss the benefits of using pattern search methods in the optimization of the BAO problem.
\end{abstract}

Keywords: IMRT, Radiotherapy, Beam Angle Optimization, Fluence Map Optimization, Derivative-free Optimization

\section{Introduction}

The goal of radiation therapy is to deliver a dose of radiation to the cancerous region to sterilize the tumor minimizing the damages on the surrounding healthy organs and tissues. Radiation therapy is delivered with the patient immobilized on a couch that can rotate. Typically, radiation is generated by a linear accelerator mounted on a gantry that can rotate along a central axis. The

\footnotetext{
*Corresponding author. Tel.: +351 239 851040; fax: +351 239824692.

Email addresses: hrocha@mat.uc.pt (H. Rocha), joana@fe.uc.pt (J.M. Dias), brigida@ua.pt (B.C. Ferreira), mclopes@ipocoimbra.min-saude.pt (M.C. Lopes)
} 
rotation of the couch combined with the rotation of the gantry allows radiation from almost any angle around the tumor. Many authors consider non-coplanar angles $[1,2,3,4,5]$. However, despite the fact that almost every angle is possible for radiation delivery, the use of coplanar angles is predominant. This is a way to simplify an already complex problem, and the angles considered lay in the plane of the rotation of the gantry around the patient. Regardless the evidence presented in the literature that appropriate radiation beam incidence directions can lead to a plan's quality improvement $[5,6]$, in clinical practice, most of the time, the number of beam angles is assumed to be defined a priori by the treatment planner and the beam directions are still manually selected by the treatment planner that relies mostly on his experience.

An important type of radiation therapy is intensity modulated radiation therapy (IMRT), where the radiation beam is modulated by a multileaf collimator. Multileaf collimators enable the transformation of the beam into a grid of smaller beamlets of independent intensities. Here, we will consider IMRT optimization problems using coplanar angles and will assume that the number of beam angles is defined a priori by the treatment planner.

A common way to solve the inverse planning in IMRT optimization problems is to use a beamlet-based approach. This approach leads to a large-scale programming problem. Due to the complexity of the whole optimization problem, many times the treatment planning is divided into three smaller problems which can be solved sequentially: BAO problem, fluence map optimization (FMO) problem, and leaf sequencing problem. That division has the consequence of causing a plan quality deterioration arising from the transition between the intensity problem and the realization problem [7]. Most of the efforts in the IMRT optimization community have been devoted at optimizing beamlet intensities [8]. Comparatively fewer research effort has been directed to the optimization of beam angles [9].

Except for rare exceptions, where the BAO problem is addressed as a nonconvex nonlinear problem (see, e.g., [8]), for the vast majority of previous work on beam angle optimization, the continuous $\left[0^{\circ}, 360^{\circ}\right]$ gantry angles are discretized into equally spaced beam directions with a given angle increment, such as 5 or 10 degrees, where exhaustive searches are performed directly or guided by a variety of different heuristics including simulated annealing [3, 10, 11], genetic algorithms $[9,12,13,14]$, particle swarm optimization [15] or other heuristics incorporating a priori knowledge of the problem. Although those global heuristics can avoid local optima theoretically, globally optimal or even clinically better solutions can not be obtained without a large number of objective function evaluations. Another common alternative is scoring methods, where scores are assigned to beam angles based on geometric and dosimetric information [16]. Despite the fact that these methods reduce the computational time, they have the drawback of ignoring the inter-relationship between beam angles by calculating dosimetric parameters from a single incident beam angle plan. Set covering and vector quantization are two other single-step techniques used. A comparison of all those methodologies is presented in [17] leading to the conclusion that these techniques are very similar and intertwined even knowing 
that their clinical perspectives may differ.

The concept of beam's eye view has been a popular approach to address the BAO problem as well $[3,18,19]$. The concept is similar to a bird's eye view, where the object being viewed is the tumor as seen from a beam. The bigger the area of the tumor and the smaller the area of the surrounding organs is seen by the beam, the better candidate the beam is to be used in the treatment plan. Other approaches include the projection of the surrounding organs into the tumor. Pugachev and Xing [20] present a computer assisted selection of coplanar angles based on a variation of the beam's eye view concept. Many other attempts to address the BAO problem can be found in literature. Ehrgott et al. [9] discuss a mathematical framework that unifies the approaches found in literature. Aleman et al. [2] propose a response surface approach and include non-coplanar angles in beam orientation optimization. Lim and Cao [21] propose an approach that consists of two sequential phases: branch-and-prune and local neighborhood search. Lee et al. [22] suggests a mixed integer programming (MIP) approach for simultaneously determining an optimal intensity map and optimal beam angles for IMRT delivery. Schreibmann et al. [23] propose a hybrid multiobjective evolutionary optimization algorithm for IMRT inverse planning and apply it to the optimization of the number of incident beams, their orientations and intensity profiles. Other approaches include maximal geometric separation of treatment beams [5] or gradient searches [8].

Here, similarly to $[1,2,5,8,13,23,24,25]$, we will use the optimal solution of the FMO problem to drive our BAO problem. Most of the previous BAO studies are based on a variety of scoring methods or approximations to the FMO to gauge the quality of the beam angle set. When the BAO problem is not based on the optimal FMO solutions, the resulting beam angle set has no guarantee of optimality and has questionable reliability since it has been extensively reported that optimal beam angles for IMRT are often non-intuitive [26]. Obtaining the optimal solution for a beam angle set is time costly and even if only one beam angle is changed in that set, a complete dose computation is required in order to compute and obtain the corresponding optimal FMO solution. To minimize this time issue, methods that require few function value evaluations should be used to tackle the BAO problem. Additionally, the BAO problem is quite difficult since it is a highly non-convex optimization problem with many local minima [8]. Therefore, methods that avoid being easily trapped in local minima should be used as well.

The objective of this paper is to introduce a new approach for the resolution of the BAO problem, using pattern search methods to tackle this highly non-convex optimization problem. Pattern search methods gather the two characteristics enumerated in the previous paragraph, making them suited to address the $\mathrm{BAO}$ problem. The paper is organized as follows. In the next section we describe the BAO problem formulation and the coupled FMO problem formulation. Section 3 briefly presents the pattern search methods framework used. Clinical examples of head-and-neck cases used in the computational tests are presented in section 4 . Section 5 presents the obtained results. In the last section we have the conclusions and future work. 


\section{Beam angle optimization problem}

In order to model the $\mathrm{BAO}$ problem as a mathematical programming problem, a quantitative measure to compare the quality of different sets of beam angles is required. For the reasons presented in section 1, our approach for modeling the BAO problem uses the optimal solution value of the FMO problem as the measure of the quality of a given beam angle set. Thus, we will present the formulation of the $\mathrm{BAO}$ problem followed by the formulation of the FMO problem we used.

\subsection{BAO model}

Let us consider $k$ to be the fixed number of (coplanar) beam directions, i.e., $k$ beam angles are chosen on a circle around the CT-slice of the body that contains the isocenter (typically the center of mass of the tumor). A basic formulation for the BAO problem is obtained by selecting an objective function such that the best set of beam angles is obtained for the function's minimum:

$$
\begin{array}{ll}
\min & f\left(\theta_{1}, \ldots, \theta_{k}\right) \\
\text { s.t. } & \theta_{1}, \ldots, \theta_{k} \in \Theta, \quad \text { where } \Theta \text { is the set of all possible angles. }
\end{array}
$$

Typically, the BAO problem is formulated as a combinatorial optimization problem in which a specified number of beam angles is to be selected among a beam angle candidate pool. The continuous $\left[0^{\circ}, 360^{\circ}\right]$ gantry angles are generally discretized into equally spaced directions with a given angle increment, such as 5 or 10 degrees originating $\Theta=\Theta_{1}^{k}, \Theta_{1}=\{0,5, \ldots, 355\}$ or $\Theta=\Theta_{2}^{k}$, $\Theta_{2}=\{0,10, \ldots, 350\}$, respectively. We could think in all possible combinations of $k$ beam angles as an exhaustive global search method. However, this requires an enormous amount of time to calculate and compare all dose distributions for all possible angle combinations. For example if we choose $k=5$ angles out of 72 candidate beam angles $\left(\Theta_{1}\right)$, there are $C_{5}^{72}=13,991,544$ combinations. By decreasing the number of candidate beam angles to $36\left(\Theta_{2}\right)$, the number of different combinations is still $C_{5}^{36}=376,992$, requiring an enormous amount of time to compare all the resulting plans regardless the measure considered in (1). Therefore, an exhaustive search of a large-scale combinatorial problem is considered to be too slow and inappropriate for a clinical setting. Many heuristics and meta-heuristics have been presented as an attempt to reduce the number of combinations to compare. However, most require a prohibitive number of function evaluations when the measure considered is the optimal value of the FMO problem.

We will consider a different approach for the formulation of the BAO problem. All continuous $\left[0^{\circ}, 360^{\circ}\right]$ gantry angles will be considered instead of a discretized sample. Since the angle $-5^{\circ}$ is equivalent to the angle $355^{\circ}$ and the angle $365^{\circ}$ is the same as the angle $5^{\circ}$, we can avoid a bounded formulation and 
consider the following unconstrained BAO model:

$$
\begin{array}{ll}
\min & f\left(\theta_{1}, \ldots, \theta_{k}\right) \\
\text { s.t. } & \theta_{1}, \ldots, \theta_{k} \in \mathbb{R}^{k}(=\Theta) .
\end{array}
$$

The objective $f\left(\theta_{1}, \ldots, \theta_{k}\right)$ that measures the quality of the set of beam directions $\theta_{1}, \ldots, \theta_{k}$ is chosen, in practice, in many different ways, expressing different criteria. Such functions may have numerous local optima, which increase the difficulty of obtaining a good global solution. Thus, the choice of the solution method becomes a critical aspect for obtaining a good solution. Our formulation was mainly motivated by the ability of using a class of solution methods that we consider to be suited to successfully address the BAO problem: pattern search methods. The FMO model used is presented next.

\subsection{FMO model}

For a given beam angle set, an optimal IMRT plan is obtained by solving the FMO problem - the problem of determining the optimal beamlet weights for the fixed beam angles. Many mathematical optimization models and algorithms have been proposed for the FMO problem, including linear models [27], mixed integer linear models [28], nonlinear models [29], and multiobjective models [30].

Radiation dose distribution deposited in the patient, measured in Gray (Gy), needs to be assessed accurately in order to solve the FMO problem, i.e., to determine optimal fluence maps. Each structure's volume is discretized in voxels (small volume elements) and the dose is computed for each voxel using the superposition principle, i.e., considering the contribution of each beamlet. Typically, a dose matrix $\mathrm{D}$ is constructed from the collection of all beamlet weights, by indexing the rows of $\mathrm{D}$ to each voxel and the columns to each beamlet, i.e., the number of rows of matrix D equals the number of voxels $(V)$ and the number of columns equals the number of beamlets $(N)$ from all beam directions considered. Therefore, using matrix format, we can say that the total dose received by the voxel $i$ is given by $\sum_{j=1}^{N} D_{i j} w_{j}$, with $w_{j}$ the weight of beamlet $j$. Usually, the total number of voxels considered reaches the tens of thousands, thus the row dimension of the dose matrix is of that magnitude. The size of $D$ originates large-scale problems being one of the main reasons for the difficulty of solving the FMO problem.

Here, we will use a convex penalty function voxel-based nonlinear model [24]. In this model, each voxel is penalized according to the square difference of the amount of dose received by the voxel and the amount of dose desired/allowed for the voxel. This formulation yields a quadratic programming problem with only linear non-negativity constraints on the fluence values [27]:

$$
\begin{array}{ll}
\min _{w} & \sum_{i=1}^{V} \frac{1}{v_{S}}\left[\underline{\lambda}_{i}\left(T_{i}-\sum_{j=1}^{N} D_{i j} w_{j}\right)_{+}^{2}+\bar{\lambda}_{i}\left(\sum_{j=1}^{N} D_{i j} w_{j}-T_{i}\right)_{+}^{2}\right] \\
\text { s.t. } & w_{j} \geq 0, j=1, \ldots, N,
\end{array}
$$


where $T_{i}$ is the desired dose for voxel $i, \underline{\lambda}_{i}$ and $\bar{\lambda}_{i}$ are the penalty weights of underdose and overdose of voxel $i$, and $(\cdot)_{+}=\max \{0, \cdot\}$. Although this formulation allows unique weights for each voxel, similarly to the implementation in [24], weights are assigned by structure only so that every voxel in a given structure has the weight assigned to that structure divided by the number of voxels of the structure $\left(v_{S}\right)$. This nonlinear formulation implies that a very small amount of underdose or overdose may be accepted in clinical decision making, but larger deviations from the desired/allowed doses are decreasingly tolerated [24].

The optimal set of beam angles depends on the penalty weight values of the objective function selected. For example, for a head-and-neck cancer case, a higher penalty weight on the parotid objective results in a set of beams which will enhance better parotid sparing. For multi-objective IMRT optimization [30], each Pareto optimal treatment plan will have a distinct set of optimal beam angles. For traditional trial-and-error parameter tuning for IMRT planning, it is not clear how BAO should be incorporated into the planning process [8]. The penalty weights were manually selected to obtain acceptable treatment plans.

Other models used before for BAO include convex penalty function structurebased approaches [25] and a variety of linear approaches [8,31]. It is beyond the scope of this study to discuss if this formulation of the FMO problem is preferable to others. The FMO model is used as a black-box function. The conclusions drawn regarding BAO coupled with this nonlinear model are valid also if different FMO formulations are considered.

\section{Pattern search methods}

Pattern search methods are directional direct search methods that belong to a broader class of derivative-free optimization methods (see [32] for a detailed overview of derivative-free optimization methods), such that iterate progression is solely based on a finite number of function evaluations in each iteration, without explicit or implicit use of derivatives. We will briefly describe pattern search methods for unconstrained optimization problems of the form

$$
\begin{array}{ll}
\min & f(x) \\
\text { s.t. } & x \in \mathbb{R}^{n},
\end{array}
$$

where the decision vector $x$ is used as input into the black-box function $f$. This type of resolution method is suited for the beam angle problem formulation presented in (2). Similar to other derivative-free optimization methods, when minimizing non-convex functions with a large number of local minima, pattern search methods have the ability to avoid being trapped by the closest local minima of the starting iterate, and find a local minimum in a lowest region, i.e., in a region with lower function values.

The pattern search methods framework [33] is the class of the most used and implemented directional direct search methods. Each iteration is composed of a search step and a poll step. Pattern search methods framework as described in [33] or later in [34] is presented next. 
Pattern search methods are iterative methods generating a sequence of iterates $\left\{x_{k}\right\}$ using positive bases or positive spanning sets and moving in the direction that would produce a function decrease. Therefore, in order to describe pattern search methods we need to describe the notions and motivations for the use of positive bases.

The positive span (strictly speaking we should say nonnegative) of a set of vectors $v_{1} \cdots v_{m} \in \mathbb{R}^{n}$ is the convex cone

$$
L_{0}^{+}\left(v_{1}, \ldots, v_{m}\right)=\left\{v \in \mathbb{R}^{n}: v=\alpha_{1} v_{1}+\cdots+\alpha_{m} v_{m}, \quad \alpha_{i} \geq 0, i=1, \ldots, m\right\} .
$$

The set $\left\{v_{1}, \ldots, v_{m}\right\}$ is said to be positively dependent if one of the vectors is in the convex cone positively spanned by the remaining vectors, i.e., if one of the vectors is a positive combination of the others. Otherwise the set is positively independent.

A positive basis for $\mathbb{R}^{n}$ is a positively independent set of $\mathbb{R}^{n}$ whose positive span is $\mathbb{R}^{n}$. A positive basis for $\mathbb{R}^{n}$ can also be defined as a set of nonzero vectors of $\mathbb{R}^{n}$ whose positive combinations span $\mathbb{R}^{n}$, but no proper set (strict subset) does.

It can be shown that a positive basis for $\mathbb{R}^{n}$ contains at least $n+1$ vectors and cannot contain more than $2 n$ [35]. Positive basis with $n+1$ and $2 n$ elements are referred to as minimal and maximal positive basis, respectively. In Figure 1 we have an illustration of a minimal positive basis ( $[I-e]$, with $I$ being the identity matrix of dimension 2 and $\left.e=\left[\begin{array}{ll}1 & 1\end{array}\right]^{\top}\right)$ and a maximal positive basis $([I-I])$.

The following theorem [35] presents three necessary and sufficient characterizations for positive bases and are the motivation for directional direct search methods:

Theorem 3.1. Let $\left\{v_{1}, \ldots, v_{m}\right\}$, with $v_{i} \neq 0$ for all $i \in\{1, \ldots, m\}$, span $\mathbb{R}^{n}$. Then the following are equivalent:

(i) $\left\{v_{1}, \ldots, v_{m}\right\}$ is a positive basis for $\mathbb{R}^{n}$.

(ii) For every $i=1, \ldots, m,-v_{i}$ is in the convex cone positively spanned by the remaining $v_{j}, j \neq i$.

(iii) There exist real scalars $\alpha_{1}, \ldots, \alpha_{m}$ with $\alpha_{i}>0, i \in\{1, \ldots, m\}$, such that $\sum_{i=1}^{m} \alpha_{i} v_{i}=0$.

(iv) For every nonzero vector $b \in \mathbb{R}^{n}$, there exists an index $i$ in $\{1, \ldots, m\}$ for which $b^{\top} v_{i}>0$.

The characterization given by Theorem 3.1.iv (considering $b=-\nabla f\left(x_{k}\right)$ ) implies that, unless the current iterate is at a stationary point, there is always a vector $v_{i}$ in a positive basis (or positive spanning set) that is a descent direction, i.e., $-\nabla f\left(x_{k}\right)^{\top} v_{i}>0$. In other words, the previous inequality means that there is always a vector $v_{i}$ in a positive basis that forms an acute angle with $-\nabla f\left(x_{k}\right)$. Therefore, $v_{i}$ is a descent direction in the sense that there is an $\alpha>0$ such that 


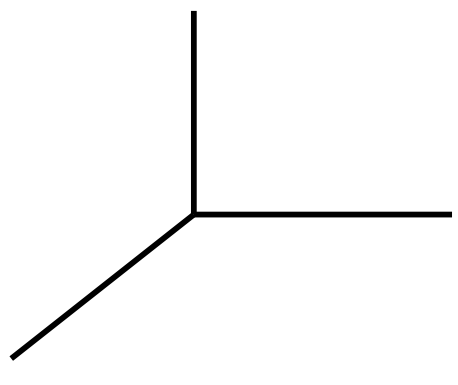

(a)

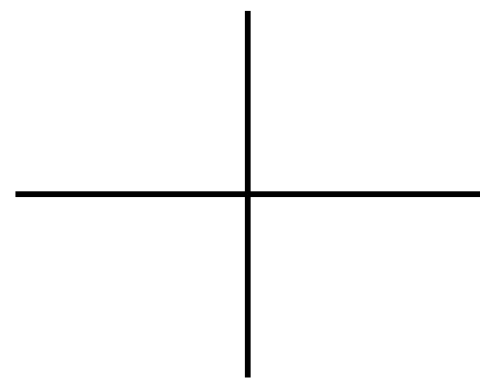

(b)

Figure 1: Examples of minimal (a) and maximal (b) positive bases in $\mathbb{R}^{2}$.

$f\left(x_{k}+\alpha v_{i}\right)<f\left(x_{k}\right)$. This is the core of directional direct search methods and in particular of pattern search methods.

Pattern search methods are iterative methods generating a sequence of nonincreasing iterates $\left\{x_{k}\right\}$. Given the current iterate $x_{k}$, at each iteration $k$, the next point $x_{k+1}$ is chosen from a finite number of candidates on a given mesh $M_{k}$ aiming to provide a decrease on the objective function: $f\left(x_{k+1}\right)<f\left(x_{k}\right)$. In order to define the mesh $M_{k}$, let us denote by $\mathcal{V}$ the matrix whose columns correspond to the $|\mathcal{V}|$ vectors forming a positive spanning set. The mesh at iteration $k$ is then defined as

$$
M_{k}=\left\{x_{k}+\Delta_{k} \mathcal{V} z: z \in \mathbb{Z}_{+}^{|\mathcal{V}|}\right\},
$$

where $\Delta_{k}$ is the mesh parameter and $\mathbb{Z}_{+}$is the set of nonnegative integers.

Pattern search methods consider two phases at every iteration. The first phase, or step, consists of a finite search on the mesh, with the goal of finding a new iterate that decreases the value of the objective function at the current iterate. This step, called the search step, has the flexibility to use any strategy, method or heuristic, or take advantage of a priori knowledge of the problem at hand, as long as it searches only a finite number of points in the mesh. If the search step is unsuccessful, a second phase or step, called the poll step, is performed around the current iterate with the goal of decreasing the objective function.

The poll step follows stricter rules and appeals to the concepts of positive bases. In this step the candidate for a new iterate $x_{k+1}$ is chosen in the mesh neighborhood around $x_{k}$

$$
\mathcal{N}\left(x_{k}\right)=\left\{x_{k}+\Delta_{k} v: \text { for all } v \in V\right\} \subset M_{k},
$$

where $V$ is a positive basis (or positive spanning set) chosen from the finite set $\mathcal{V}$.

We have now all the ingredients to describe pattern search methods. 
Algorithm 3.1 (Pattern search methods).

0. Initialization Choose $x_{0} \in \mathbb{R}^{n}, \Delta_{0}>0$, and a positive spanning set $V \subset \mathcal{V}$. Choose a rational number $\tau>1$ and an integer number $m_{\max } \geq 1$. Set $k=0$.

1. Search step (in current mesh) Try to obtain a decrease of the objective function value at $x_{k}$ by evaluating $f$ at a finite number of points in $M_{k}$. If $x_{k+1} \in M_{k}$ is found satisfying $f\left(x_{k+1}\right)<f\left(x_{k}\right)$, go to step 3 , expanding $M_{k}$ (search step and iteration are declared successful).

2. Poll step (in mesh neighborhood) This step is only performed if the search step is unsuccessful. If $f\left(x_{k}\right) \leq f(x)$ for every $x$ in the mesh neighborhood $\mathcal{N}\left(x_{k}\right)$, go to step 4 , shrinking $M_{k}$ (poll step and iteration are declared unsuccessful). Otherwise, choose a point $x_{k+1} \in \mathcal{N}\left(x_{k}\right)$ such that $f\left(x_{k+1}\right)<f\left(x_{k}\right)$ and go to step 3, expanding $M_{k}$ (poll step and iteration are declared successful).

3. Mesh expansion (at successful iterations) Let $\Delta_{k+1}=\tau^{m_{k}^{+}} \Delta_{k}$, with $0 \leq m_{k}^{+} \leq m_{\max }$. Increase $k$ by one, and return to step 1 for a new iteration.

4. Mesh reduction (at unsuccessful iterations) Let $\Delta_{k+1}=\tau^{m_{k}^{-}} \Delta_{k}$, with $-m_{\max } \leq m_{k}^{-} \leq-1$. Increase $k$ by one, and return to step 1 for a new iteration.

The search step provides the flexibility for a global search since it allows searches away from the neighborhood of the current iterate, and influences the quality of the local minimizer or stationary point found by the method. The poll step is applied when the search step fails to produce a better point. The poll step attempts to perform a local search in a mesh neighborhood that, for a sufficiently small mesh parameter $\Delta_{k}$, is guaranteed to provide a function reduction, unless the current iterate is at a stationary point (a fact that can be inferred by Theorem 3.1.iv with $b=-\nabla f\left(x_{k}\right)$ ). So, if the poll step also fails, the mesh parameter $\Delta_{k}$ must be decreased. The most common choice for the mesh parameter update is to half the mesh parameter at unsuccessful iterations and to keep it or double it at successful ones. Note that if the initial mesh parameter is a power of $2,\left(\Delta_{0}=2^{p}, p \in \mathbb{N}\right)$, and the initial point is a vector of integers, using this common mesh update, all iterates will be a vector of integers until the mesh parameter size becomes inferior to 1 . This possibility is rather interesting for our BAO problem at hand.

Typically, the stopping criteria of the pattern search methods is based either on the maximum number of function value evaluations allowed or in convergence criteria related with the mesh size. Provided the following assumption is made on the mesh: each column $i$ of $\mathcal{V}$ is given by $G \bar{z}_{i}$, where $G \in \mathbb{R}^{n \times n}$ is a nonsingular generating matrix and $\bar{z}_{i}$ is an integer vector in $\mathbb{Z}^{n}$, pattern search methods share the following convergence result [34]: 
Theorem 3.2. Suppose that the level set $L\left(x_{0}\right)=\left\{x \in \mathbb{R}^{n}: f(x) \leq f\left(x_{0}\right)\right\}$ is compact and that $f$ is continuously differentiable in an open set containing $L\left(x_{0}\right)$. Then

$$
\liminf _{k \longrightarrow+\infty}\left\|\nabla f\left(x_{k}\right)\right\|=0,
$$

and there exists at least one limit point $x_{*}$ such that $\nabla f\left(x_{*}\right)=0$.

Furthermore, if $\lim _{k \longrightarrow+\infty} \Delta_{k}=0,\left\|x_{k+1}-x_{k}\right\| \leq C \Delta_{k}$ for some constant $C>0$ independent of the iteration counter $k$, and $x_{k+1}=\operatorname{argmin}_{x \in \mathcal{N}\left(x_{k}\right)} f(x)$ in the poll step, then

$$
\lim _{k \longrightarrow+\infty}\left\|\nabla f\left(x_{k}\right)\right\|=0,
$$

and every limit point $x_{*}$ satisfies $\nabla f\left(x_{*}\right)=0$.

The results of Theorem 3.2 concern the ability of pattern search methods to converge globally, i.e., from arbitrary points, to local minimizers candidates. We recall, despite the inexistence of any supporting theory, that due to their blindness caused by the nonuse of derivatives, and also by the flexibility of the search step to incorporate global search procedures while the poll step continues to assure convergence to local minima, numerical evidence about the capability of pattern search methods to compute global minimizers has been reported see, e.g., $[34,36]$.

To address the BAO problem, efficiency on the number of function value computations is of the utmost importance. Therefore, the number of trial points in the search step should be minimalist, and guided by some physical or biological meaning. On the other hand, when the search step fails to obtain a decrease on the function value, polling should also be oriented in order to further reduce the number of function value evaluations (at least for successful iterations). Recently, the efficiency of pattern search methods improved significantly by reordering the poll directions according to descent indicators built from simplex gradients [37]. Adding to that, the search step was provided with the use of minimum Frobenius norm quadratic models to be minimized within a trust region, which can lead to a significant improvement of direct search for smooth, piecewise smooth, and noisy problems [36]. For driving the resolution of the BAO problem, we will use the last version of SID-PSM $[36,37]$ which is a MATLAB [38] implementation of the pattern search methods that incorporate the referred improvements for the search and the poll steps.

The benefits of using pattern search methods in the optimization of the BAO problem are illustrated using a set of clinical examples of head-and-neck cases that are presented next.

\section{Head-and-neck clinical examples}

Four clinical examples of retrospective treated cases of head-and-neck tumors at the Portuguese Institute of Oncology of Coimbra are used to test the 


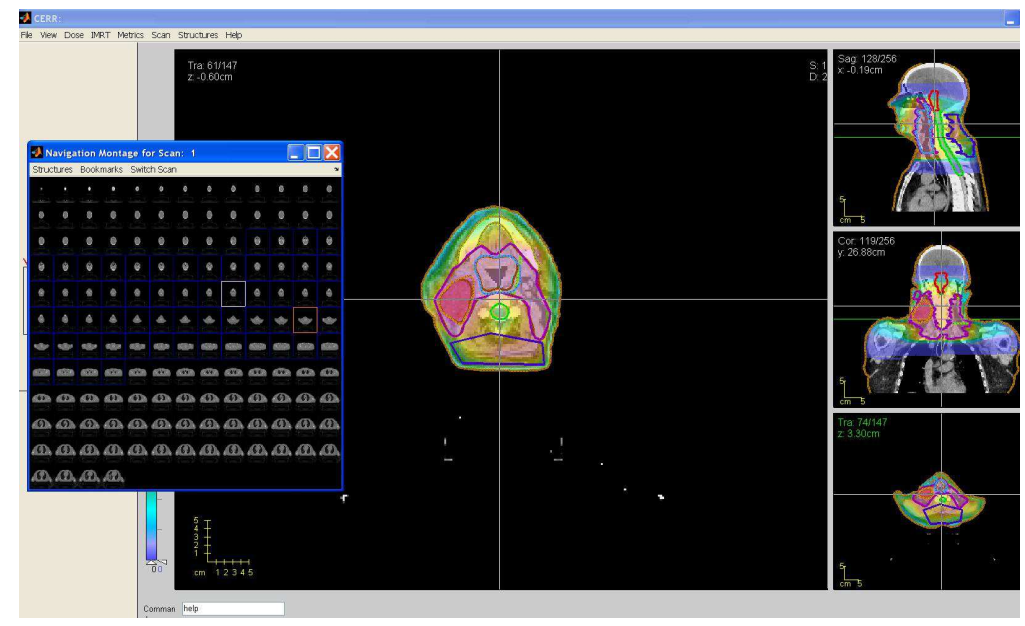

Figure 2: Structures considered in the IMRT optimization visualized in CERR.

pattern search methods framework proposed. The selected clinical examples were signalized at IPOC as complex cases where proper target coverage and organ sparing, in particular parotid sparing, proved to be difficult to obtain with the typical 7-beam equispaced coplanar treatment plans. The patients' CT sets and delineated structures were exported via Dicom RT to a freeware computational environment for radiotherapy research (see Figure 2). Since the head-and-neck region is a complex area where, e.g., the parotid glands are usually in close proximity to or even overlapping with the target volume, careful selection of the radiation incidence directions can be determinant to obtain a satisfying treatment plan.

The spinal cord and the brainstem are some of the most critical organs at risk (OARs) in the head-and-neck tumor cases. These are serial organs, i.e., organs such that if only one subunit is damaged, the whole organ functionality is compromised. Therefore, if the tolerance dose is exceeded, it may result in functional damage to the whole organ. Thus, it is extremely important not to exceed the tolerance dose prescribed for these type of organs. Other than the spinal cord and the brainstem, the parotid glands are also important OARs. The parotid gland is the largest of the three salivary glands. A common complication due to parotid glands irradiation is xerostomia (the medical term for dry mouth due to lack of saliva). This decreases the quality of life of patients undergoing radiation therapy of head-and-neck, causing difficulties to swallow. The parotids are parallel organs, i.e., if a small volume of the organ is damaged, the rest of the organ functionality may not be affected. Their tolerance dose depends strongly on the fraction of the volume irradiated. Hence, if only a small fraction of the organ is irradiated the tolerance dose is much higher than if a larger fraction is irradiated. Thus, for these parallel structures, the organ mean dose is generally used instead of the maximum dose as an objective for inverse 


\begin{tabular}{lccc}
\hline Structure & Mean dose & Max dose & Prescribed dose \\
\hline Spinal cord & - & $45 \mathrm{~Gy}$ & - \\
Brainstem & - & $54 \mathrm{~Gy}$ & - \\
Left parotid & $26 \mathrm{~Gy}$ & - & - \\
Right parotid & $26 \mathrm{~Gy}$ & - & - \\
PTV1 & - & - & $70.0 \mathrm{~Gy}$ \\
PTV2 & - & - & $59.4 \mathrm{~Gy}$ \\
Body & - & $80 \mathrm{~Gy}$ & - \\
\hline
\end{tabular}

Table 1: Prescribed doses for all the structures considered for IMRT optimization.

planning optimization.

In general, the head-and-neck region is a complex area to treat with radiotherapy due to the large number of sensitive organs in this region (e.g., eyes, mandible, larynx, oral cavity, etc.). For simplicity, in this study, the OARs used for treatment optimization were limited to the spinal cord, the brainstem and the parotid glands.

The tumor to be treated plus some safety margins is called planning target volume (PTV). For the head-and-neck cases in study it was separated in two parts with different prescribed doses: PTV1 and PTV2. The prescription dose for the target volumes and tolerance doses for the organs at risk considered in the optimization are presented in Table 1.

The parotid glands are in close proximity to or even overlapping with the PTV which helps explaining the difficulty of parotid sparing. Adequate beam directions can help on the overall optimization process and in particular in parotid sparing.

\section{Results}

The pattern search methods framework was tested using a set of four clinical examples of retrospective treated cases of head-and-neck tumors at the Portuguese Institute of Oncology of Coimbra (IPOC). A typical head-and-neck treatment plan consists of radiation delivered from 5 to 9 equally spaced coplanar orientations around the patient. Treatment plans with 7 equispaced coplanar beams were used at IPOC and are commonly used in practice to treat head-and-neck cases [2]. Therefore, treatment plans of 7 coplanar orientations, denoted 7 PSM, are obtained using the pattern search method framework and compared with 7-beam equispaced coplanar treatment plans, denoted 7 equi. Treatment plans of 5 coplanar orientations, denoted 5 PSM, were also obtained using the pattern search method framework and compared with the traditional 7-beam equispaced coplanar treatment plans. The objective of these comparisons is twofold. First, to demonstrate that using the same number of beam angles, the quality of the plan can be improved with our BAO approach. Second, to highlight that it is possible to reduce the number of beam angles and 
still obtain quality treatment plans. Obtaining acceptable treatment plans that require fewer beams than in current practice may reduce the length of treatment time, which is an important clinical aspect in IMRT.

In order to facilitate convenient access, visualization and analysis of patient treatment planning data, as well as dosimetric data input for treatment plan optimization research, the computational tools developed within MATLAB and CERR - computational environment for radiotherapy research [39] are used widely for IMRT treatment planning research. The ORART - operations research applications in radiation therapy [40] collaborative working group developed a series of software routines that allow access to influence matrices, which provide the necessary dosimetry data to perform optimization in IMRT. CERR was elected as the main software platform to embody our optimization research.

Our tests were performed on a 2.66Ghz Intel Core Duo PC with 3 GB RAM. We used CERR 3.2.2 version and MATLAB 7.4.0 (R2007a). The dose was computed using CERR's pencil beam algorithm (QIB). For each of the four headand-neck cases, the sample rate used for Body was 32 and for the remaining structures was 4 , resulting in 20,874 to 24,158 voxels and 948 to 1,283 beamlets for the 7-beam equispaced coplanar treatment plans. An automatized procedure for dose computation for each given beam angle set was developed, instead of the traditional dose computation available from IMRTP module accessible from CERR's menubar. This automatization of the dose computation was essential for integration in our BAO algorithm. To address the convex nonlinear formulation of the FMO problem we used a trust-region-reflective algorithm (fmincon) of MATLAB 7.4.0 (R2007a) Optimization Toolbox. The last version of SID-PSM was used as our pattern search methods framework. Since we want to improve the quality of the 7 equi treatment plans, the 7 equispaced coplanar beam angles were considered as initial point for the 7-beam angle optimization process. For the 5 -beam angle optimization process we considered the 5 equispaced coplanar beam angles as initial point. The choice of this initial points and the non-increasing property of the sequence of iterates generated by SID-PSM imply that each successful iteration correspond to an effective improvement with respect to the usual equispaced beam configuration. The spanning set used was the positive spanning set $\left(\left[\begin{array}{lll}e-e & I-I\end{array}\right]\right.$, with $I$ being the identity matrix of dimension $k$ and $\left.e=\left[\begin{array}{lll}1 & \ldots & 1\end{array}\right]^{T}\right)$. Each of these directions correspond to, respectively, the rotation of all incidence directions clockwise, to the rotation of all incidence directions counter-clockwise, the rotation of each individual incidence direction clockwise, and the rotation of each individual incidence direction counter-clockwise. The initial size of the mesh parameter was set to $2^{4}=16$. Smaller powers of 2 were tested, namely 4 and 8 , with worst results and worst coverage of the whole search space. Larger powers of 2 were tested, namely 32 and 64, with similar results and coverage of the whole search space, but at the cost of more function evaluations. Since the initial points were integer vectors, all iterates will have integer values as long as the mesh parameter does not become less than 1 . Therefore, the stopping criteria adopted was the mesh parameter becoming less than 1 . 


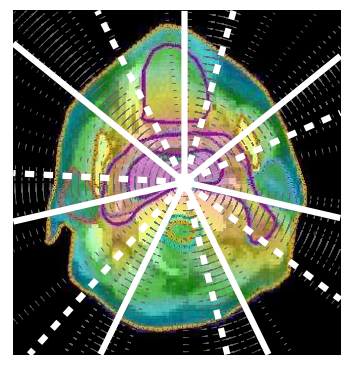

(a)

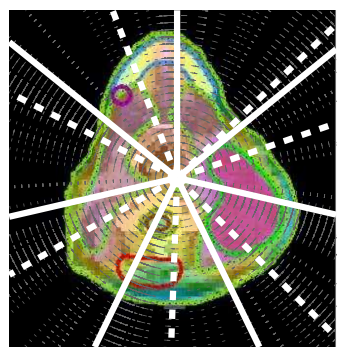

(c)

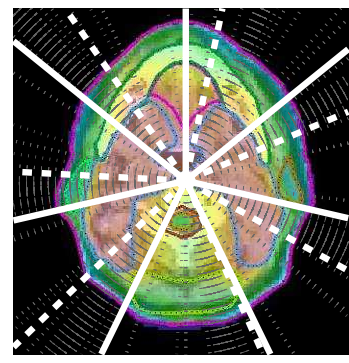

(b)

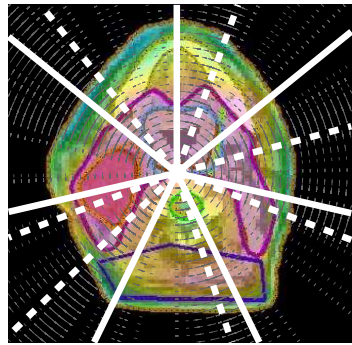

(d)

Figure 3: History of the 7-beam angle optimization process using SID-PSM for cases 1 to 4, $3(\mathrm{a})$ to $3(\mathrm{~d})$ respectively. Initial angle configuration, optimal angle configuration and intermediate angle configurations are displayed with solid, dashed and dotted lines, respectively.

The history of the 7-beam angle optimization process using SID-PSM, in terms of beam directions tested, for each case, is presented in Figure 3. By simple inspection we can verify that the sequence of iterates are reasonably well distributed by amplitude in $\mathbb{R}^{2}$, with an appropriate coverage in amplitude of all the search space. The mean variation of each obtained beam direction with respect to the initial equispaced directions is $12.9^{\circ}$. Here, we have to highlight that, BAO performed for these retrospective treated cases, using IPOC's commercial treatment planning system, obtained a mean variation of each beam direction with respect to the initial equispaced directions inferior to $4^{\circ}$, which is not clinically relevant. One of the main advantages of this pattern search methods framework is the flexibility provided by the search step, where any strategy can be applied as long as only a finite number of points is tested. This allows the insertion of previously used and tested strategies/heuristics that successfully address the BAO problem and enhance for a global search by influencing the quality of the local minimizer or stationary point found by the method. In the last version of SID-PSM, the search step computes a single trial point using 


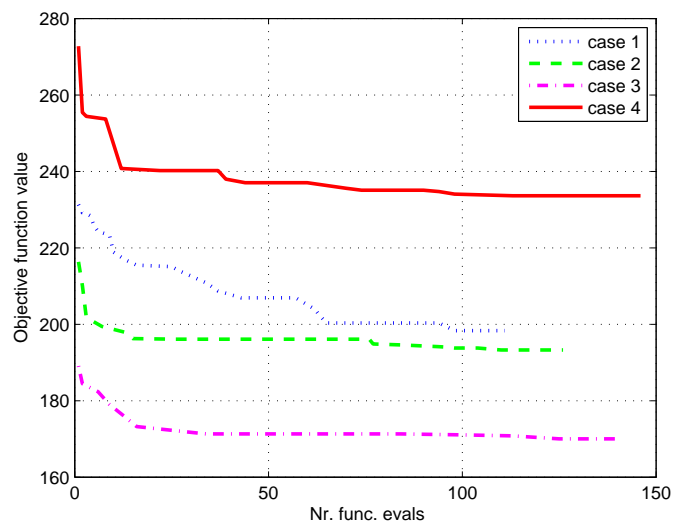

Figure 4: Objective function value history of the 7-beam angle optimization process for each head-and-neck case.

minimum Frobenius norm quadratic models to be minimized within a trust region, which enhanced a significant improvement of direct search for black-box non-smooth functions [36] similar to the BAO problem at hand. The size of the trust region is coupled to radius of the sample set. Thus, for a global search attempt, the sample points should span all the search space. However, since the BAO problem has many local minima and the number of sample points is scarce, the polynomial interpolation or regression models (usually quadratic models) used within the trust region may struggle to find the best local minima. Global strategies like particle swarm methods can be included in the search step to enhance a global search - see [41]. However, the number of function evaluations required for this type of strategies is prohibitive for obtaining an answer in a clinically acceptable time frame.

The history of the 7-beam angle optimization process using SID-PSM, presented in terms of the number of function evaluations, is shown in Figure 4. The mean decrease of the objective function was $12.4 \%$ with a mean computational time of 3.7 hours. Dose computation using QIB consumed most of the overall computational time. For different dose engines, different beamlet optimization methods and different objective function strategies, the overall computational time may be of significant different magnitude. Therefore, as important as mentioning that in average it took 3.7 hours for the method to converge, it is important to emphasize the few number of function evaluations required by pattern search methods. It is worth to highlight as well the rapid initial decrease of the FMO value. In practice, that feature can be used for obtaining quality beam directions in shorter computational times, e.g., by adopting as stopping criteria the mesh parameter becoming less than 2 or 4 instead of 1 . For the 5 -beam angle optimization process using SID-PSM, the mean decrease of the objective function was $21.8 \%$ with a mean computational time of 2.2 hours. The size of 


\begin{tabular}{|c|c|c|c|c|}
\hline Case & Target coverage & $5 P S M$ & $7 P S M$ & 7 equi \\
\hline \multirow{6}{*}{1} & PTV1 at $95 \%$ volume & $66.93 \mathrm{~Gy}$ & $67.23 \mathrm{~Gy}$ & 67.03 Gy \\
\hline & PTV1 $\%>93 \%$ of $\operatorname{Rx}(\%)$ & 98.98 & 99.65 & 99.38 \\
\hline & PTV1 $\%>110 \%$ of $\operatorname{Rx}(\%)$ & 0.00 & 0.00 & 0.00 \\
\hline & PTV2 at $95 \%$ volume & $57.53 \mathrm{~Gy}$ & $57.93 \mathrm{~Gy}$ & $57.78 \mathrm{~Gy}$ \\
\hline & PTV2 $\%>93 \%$ of $\operatorname{Rx}(\%)$ & 97.27 & 97.76 & 97.41 \\
\hline & PTV2 $\%>110 \%$ of $\operatorname{Rx}(\%)$ & 16.25 & 15.90 & 16.02 \\
\hline \multirow{6}{*}{2} & PTV1 at $95 \%$ volume & 66.37 Gy & $67.38 \mathrm{~Gy}$ & $66.73 \mathrm{~Gy}$ \\
\hline & PTV1 $\%>93 \%$ of $\operatorname{Rx}(\%)$ & 98.86 & 99.33 & 99.06 \\
\hline & PTV1 $\%>110 \%$ of $\operatorname{Rx}(\%)$ & 0.00 & 0.00 & 0.00 \\
\hline & PTV2 at $95 \%$ volume & $56.58 \mathrm{~Gy}$ & $57.28 \mathrm{~Gy}$ & $56.98 \mathrm{~Gy}$ \\
\hline & PTV2 $\%>93 \%$ of $\operatorname{Rx}(\%)$ & 96.83 & 97.29 & 96.84 \\
\hline & PTV2 $\%>110 \%$ of $\operatorname{Rx}(\%)$ & 4.36 & 4.85 & 4.34 \\
\hline \multirow{6}{*}{3} & PTV1 at $95 \%$ volume & $65.88 \mathrm{~Gy}$ & $66.64 \mathrm{~Gy}$ & $66.63 \mathrm{~Gy}$ \\
\hline & PTV1 $\%>93 \%$ of $\operatorname{Rx}(\%)$ & 97.42 & 98.64 & 98.42 \\
\hline & PTV1 $\%>110 \%$ of $\operatorname{Rx}(\%)$ & 0.00 & 0.00 & 0.00 \\
\hline & PTV2 at $95 \%$ volume & $55.89 \mathrm{~Gy}$ & $56.88 \mathrm{~Gy}$ & $56.78 \mathrm{~Gy}$ \\
\hline & PTV2 $\%>93 \%$ of $\operatorname{Rx}(\%)$ & 95.77 & 96.78 & 96.66 \\
\hline & PTV2 $\%>110 \%$ of $\operatorname{Rx}(\%)$ & 16.79 & 17.18 & 17.66 \\
\hline \multirow{6}{*}{4} & PTV1 at $95 \%$ volume & $67.43 \mathrm{~Gy}$ & $67.58 \mathrm{~Gy}$ & $67.38 \mathrm{~Gy}$ \\
\hline & PTV1 $\%>93 \%$ of $\operatorname{Rx}(\%)$ & 99.69 & 99.74 & 99.68 \\
\hline & PTV1 $\%>110 \%$ of $\operatorname{Rx}(\%)$ & 0.00 & 0.00 & 0.00 \\
\hline & PTV2 at $95 \%$ volume & $57.23 \mathrm{~Gy}$ & $57.78 \mathrm{~Gy}$ & $57.43 \mathrm{~Gy}$ \\
\hline & PTV2 $\%>93 \%$ of $\operatorname{Rx}(\%)$ & 97.81 & 98.47 & 97.99 \\
\hline & PTV $2 \%>110 \%$ of $\operatorname{Rx}(\%)$ & 11.95 & 11.52 & 11.81 \\
\hline
\end{tabular}

Table 2: Target coverage obtained by treatment plans.

the 5-beam angle optimization problem is inferior to the 7-beam angle optimization problem (less beamlets), which requires less time for dose computation for each function value evaluation, leading to shorter computational times.

Despite the improvement in FMO value, the quality of the results can be perceived considering a variety of metrics. Typically, results are judged by their cumulative dose-volume histogram (DVH). The DVH displays the fraction of a structure's volume that receives at least a given dose. Another metric usually used for plan evaluation is the volume of PTV that receives $95 \%$ of the prescribed dose. Typically, 95\% of the PTV volume is required. The occurrence of coldspots, less than $93 \%$ of PTV volume receives the prescribed dose, and the existence of hotspots, the percentage of the PTV volume that receives more than $110 \%$ of the prescribed dose, are other measures usually used to evaluate target coverage. Mean and/or maximum doses of OArs are usually displayed to verify organ sparing.

The results regarding targets coverage are presented in Table 2. We can 


\begin{tabular}{|c|c|c|c|c|c|c|c|}
\hline \multirow[b]{2}{*}{ Case } & \multirow[b]{2}{*}{ OAR } & \multicolumn{3}{|c|}{ Mean Dose (Gy) } & \multicolumn{3}{|c|}{ Max Dose (Gy) } \\
\hline & & $5 P S M$ & $7 P S M$ & 7 equi & $5 P S M$ & $7 P S M$ & 7 equi \\
\hline \multirow{4}{*}{1} & Spinal cord & - & - & - & 40.97 & 37.76 & 38.77 \\
\hline & Brainstem & - & - & - & 48.33 & 48.40 & 53.70 \\
\hline & Left parotid & 25.23 & 23.54 & 26.99 & - & - & - \\
\hline & Right parotid & 25.52 & 23.06 & 26.18 & - & - & - \\
\hline \multirow{4}{*}{2} & Spinal cord & - & - & - & 44.39 & 43.92 & 44.78 \\
\hline & Brainstem & - & - & - & 53.88 & 52.28 & 52.05 \\
\hline & Left parotid & 25.75 & 24.37 & 27.43 & - & - & - \\
\hline & Right parotid & 27.05 & 21.28 & 27.75 & - & - & - \\
\hline \multirow{4}{*}{3} & Spinal cord & - & - & - & 44.86 & 44.56 & 44.58 \\
\hline & Brainstem & - & - & - & 48.58 & 49.21 & 48.87 \\
\hline & Left parotid & 28.57 & 27.83 & 28.36 & - & - & - \\
\hline & Right parotid & 30.37 & 28.89 & 30.54 & - & - & - \\
\hline \multirow{4}{*}{4} & Spinal cord & - & - & - & 39.27 & 39.99 & 40.63 \\
\hline & Brainstem & - & - & - & 48.05 & 47.45 & 47.31 \\
\hline & Left parotid & 27.16 & 25.74 & 27.43 & - & - & - \\
\hline & Right parotid & 27.07 & 25.75 & 27.26 & - & - & - \\
\hline
\end{tabular}

Table 3: OARs sparing obtained by treatment plans.

verify that 7 PSM treatment plans consistently obtained slightly better target coverage numbers compared to 7 equi treatment plans. On the other hand, target coverage numbers are unfavorable to 5 PSM treatment plans compared to 7 equi treatment plans. Nevertheless, in average, the differences are small. Organ sparing results are shown in Table 3. All the treatment plans fulfill the maximum dose requirements for the spinal cord and the brainstem. However, as expected, the main differences reside in parotid sparing. The 7 equi treatment plans could never enhance parotid sparing while 7 PSM treatment plans always fulfill the parotid's mean dose requirements except for case 3 . In average, 7 PSM treatment plans reduced the parotid's mean dose irradiation in 2.69 Gy compared to the 7 equi treatment plans. The differences between 5 PSM treatment plans and 7 equi treatment plans, concerning parotid sparing, are small, with advantage for the 5 PSM treatment plans, namely for case 1 where the mean dose limits were achieved. DVH results illustrating the numbers presented in Tables 2 and 3 are displayed in Figures 5 and 6 . Since parotids are the most difficult organs to spare, as shown in Table 3, for clarity, the DVHs only include the targets and the parotids. The asterisks indicate $95 \%$ of PTV volumes versus $95 \%$ of the prescribed doses. In Figure 5 the comparison of 7 equi treatment plans and the 7 PSM treatment plans is displayed. We can observe that target coverage is very similar to both treatment plans while parotid sparing is clearly better for 7 PSM treatment plans. Focusing in Figure 6, where 7 equi treatment plans and 5 PSM treatment plans are compared, despite the slight 


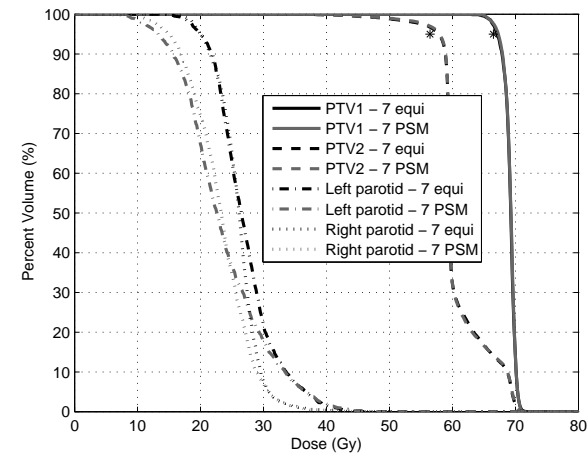

(a)

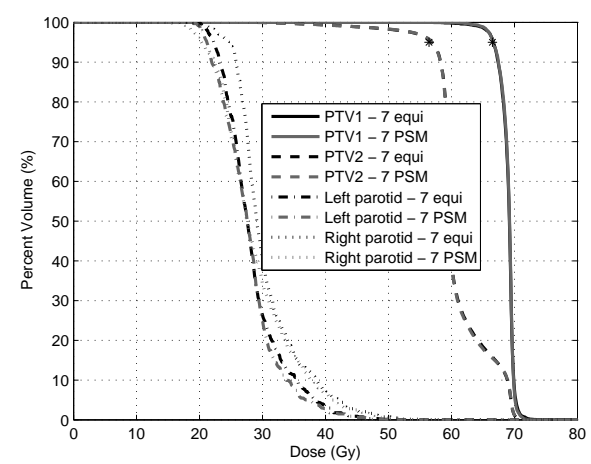

(c)

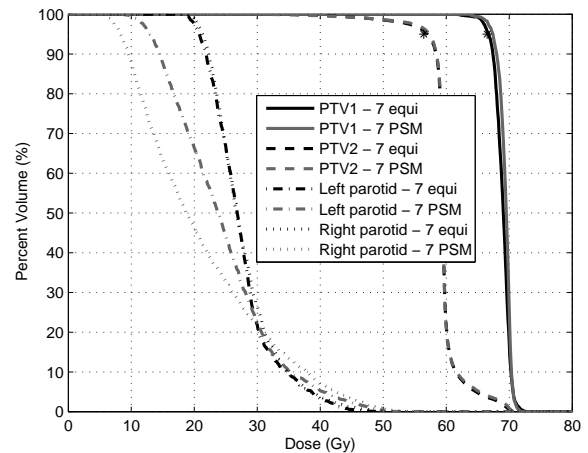

(b)

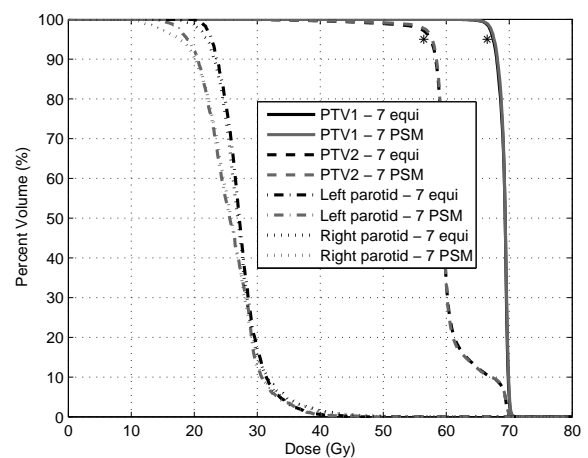

(d)

Figure 5: Cumulative dose volume histogram comparing the results obtained by 7 PSM and 7 equi for cases 1 to $4,5(\mathrm{a})$ to $5(\mathrm{~d})$ respectively.

better target coverage presented by 7 equi treatment plans and similar parotid sparing, we can verify that 5 PSM treatment plans are very competitive with 7 equi treatment plans.

The pattern search methods approach seems to be similar to neighborhood search approaches in which the neighborhood is constructed using the pattern search method. However, local neighborhood search approaches are only similar to the poll step of the pattern search methods framework. The existence of a search step with the flexibility to use any strategy, method or heuristic, or take advantage of a priori knowledge of the problem at hand, is an advantage that can be even further explored in future work. The search step provides the flexibility for a global search since it allows searches away from the neighborhood of the current iterate which perspectives a potential improvement of the method. 


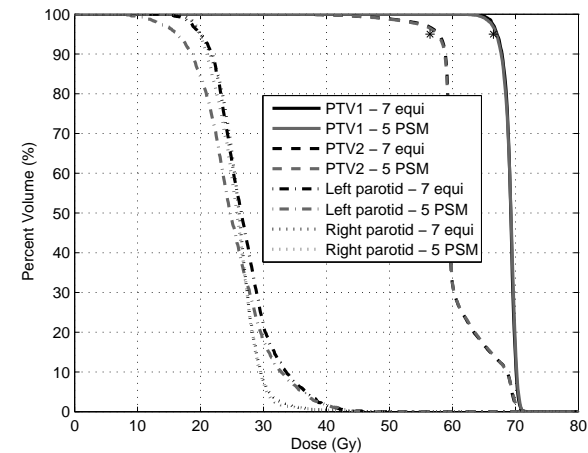

(a)

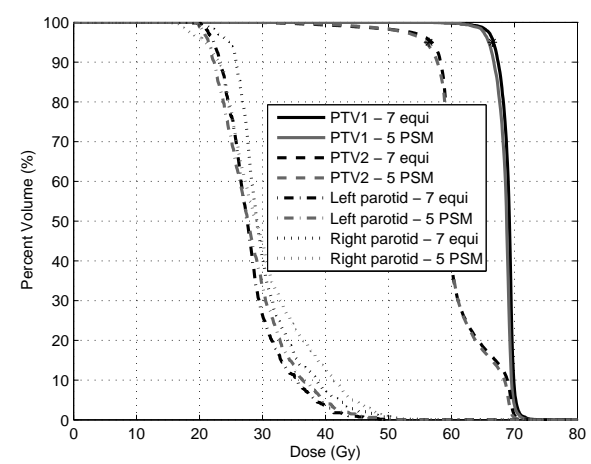

(c)

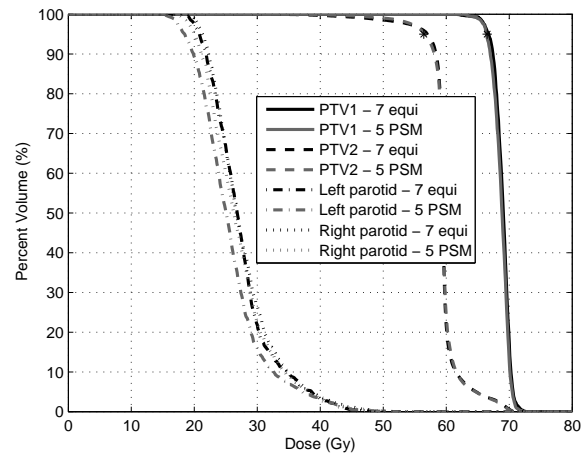

(b)

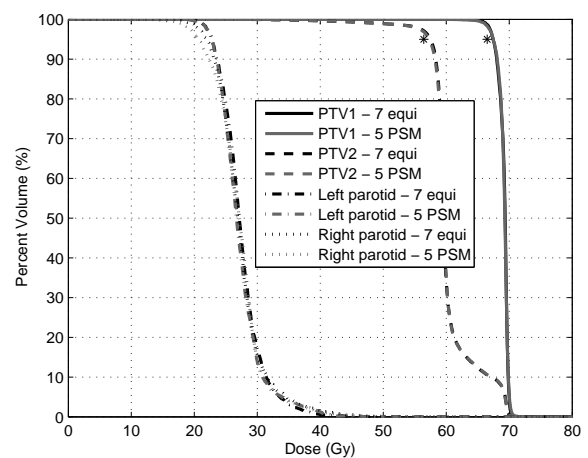

(d)

Figure 6: Cumulative dose volume histogram comparing the results obtained by 5 PSM and 7 equi for cases 1 to $4,6($ a) to $6(\mathrm{~d})$ respectively.

\section{Conclusions and future work}

The BAO problem is a continuous global highly non-convex optimization problem known to be extremely challenging and yet to be solved satisfactorily. A new approach for the resolution of the BAO problem, using a pattern search methods framework, was proposed and tested using a set of clinical head-andneck cases. We have shown that a beam angle set can be locally improved in a continuous manner using pattern search methods. Moreover, it was shown that, for head-and-neck cases, 7-beam treatment plans can be obtained with better target coverage of the typical 7-beam equidistant treatment plans and with improved organ sparing, in particular better parotid sparing. Furthermore, high-quality coplanar beam directions can be obtained and compete with equispaced beam directions in larger number within a clinically acceptable time frame. Pattern search methods framework is a suitable approach for the resolution of the non-convex BAO problem due to their structure, organized around 
two phases at every iteration. The poll step, where convergence to a local minima is assured, and the search step, where flexibility is conferred to the method since any strategy can be applied. The search step is provided with the use of minimum Frobenius norm quadratic models to be minimized within a trust region, which can lead to a significant improvement of direct search for the type of problems at hand. Nevertheless, in future work, the search step improvement will be tested with the incorporation of tailored strategies previously developed to address the BAO problem. Adding to the search step flexibility, and similarly to other derivative-free optimization methods, when minimizing non-convex functions with a large number of local minima, pattern search methods have the ability to avoid being trapped by the closest local minima of the starting iterate, and find a local minima in lowest regions. Moreover, we have to highlight the low number of function evaluations required to obtain locally optimal solutions, which is a major advantage compared to other global heuristics. This advantage should be even more relevant when considering non-coplanar directions since the number of possible directions to consider increase significantly. The efficiency on the number of function value computation is of the utmost importance, particularly when the BAO problem is modeled using the optimal values of the FMO problem.

\section{Acknowledgements}

The work of H. Rocha was supported by the European social fund and Portuguese funds from MCTES.

\section{References}

[1] V. V. Mišić, D. M. Aleman, M. B. Sharpe, Neighborhood search approaches to non-coplanar beam orientation optimization for total marrow irradiation using IMRT, Eur. J. Oper. Res. 205 (2010) 522-527.

[2] D. M. Aleman, H. E. Romeijn, J. F. Dempsey, A response surface approach to beam orientation optimization in intensity modulated radiation therapy treatment planning, INFORMS J. Comput.: Computat. Biol. Med. Appl. 21 (2009) 62-76.

[3] H. M. Lu, H. M. Kooy, Z. H. Leber, R. J. Ledoux, Optimized beam planning for linear accelerator-based stereotactic radiosurgery, Int. J. Radiat. Oncol. Biol. Phys. 39 (1997) 1183-1189.

[4] G. Meedt, M. Alber, F. Nüsslin, Non-coplanar beam direction optimization for intensity-modulated radiotherapy, Phys. Med. Biol. 48 (2003) 2999-3019.

[5] S. K. Das, L. B. Marks, Selection of coplanar or non coplanar beams using three-dimensional optimization based on maximum beam separation and minimized nontarget irradiation, Int. J. Radiat. Oncol. Biol. Phys. 38 (1997) 643-655. 
[6] H. H. Liu, M. Jauregui, X. Zhang, X. Wang, L. Dongand, R. Mohan, Beam angle optimization and reduction for intensity-modulated radiation therapy of non-small-cell lung cancers, Int. J. Radiat. Oncol. Biol. Phys. 65 (2006) $561-572$.

[7] H. Rocha, J. M. Dias, B. C. Ferreira, M. C. Lopes, Discretization of optimal beamlet intensities in IMRT: a binary integer programming approach, Math. Comput. Model. 55 (2012) 1969 - 1980.

[8] D. Craft, Local beam angle optimization with linear programming and gradient search, Phys. Med. Biol. 52 (2007) 127-135.

[9] M. Ehrgott, A. Holder, J. Reese, Beam selection in radiotherapy design, Linear Algebra Appl. 428 (2008) 1272-1312.

[10] D. Djajaputra, Q. Wu, Y. Wu, R. Mohan, Algorithm and performance of a clinical IMRT beam-angle optimization system, Phys. Med. Biol. 48 (2003) 3191-3212.

[11] T. Bortfeld, W. Schlegel, Optimization of beam orientations in radiation therapy: some theoretical considerations, Phys. Med. Biol. 38 (1993) 291-304.

[12] G.A. Ezzell, Genetic and geometric optimization of three-dimensional radiation therapy treatment planning, Med. Phys. 23 (1996) 293-305.

[13] O. C. Haas, K. J. Burnham, J. Mills, Optimization of beam orientation in radiotherapy using planar geometry, Phys. Med. Biol. 43 (1998) 2179-2193.

[14] Y. Li, J. Yao, D. Yao, Automatic beam angle selection in IMRT planning using genetic algorithm, Phys. Med. Biol. 49 (2004) 1915-1932.

[15] Y. Li, D. Yao, J. Yao, W. Chen, A particle swarm optimization algorithm for beam angle selection in intensity modulated radiotherapy planning, Phys. Med. Biol. 50 (2005) 3491-3514.

[16] W. D. D'Souza, R. R. Meyer, L. Shi, Selection of beam orientations in intensity-modulated radiation therapy using single beam indices and integer programming, Phys. Med. Biol. 49 (2004) 3465-3481.

[17] M. Ehrgott, R. Johnston, Optimisation of beam directions in intensity modulated radiation therapy planning, OR Spectrum 25 (2003) 251-264.

[18] M. Goitein, M. Abrams, D. Rowell, H. Pollari, J. Wiles, Multidimensional treatment planning: II. Beams eye-view, back projection, and projection through CT sections, Int. J. Radiat. Oncol. Biol. Phys. 9 (1983) 789-797.

[19] A. Pugachev, L. Xing, Pseudo beam's-eye-view as applied to beam orientation selection in intensity-modulated radiation therapy, Int. J. Radiat. Oncol. Biol. Phys. 51 (2001) 1361-1370. 
[20] A. Pugachev, L. Xing, Computer-assisted selection of coplanar beam orientations in intensity-modulated radiation therapy, Phys. Med. Biol. 46 (2001) $2467-2476$.

[21] G. J. Lim, W. Cao, A two-phase method for selecting IMRT treatment beam angles: Branch-and-Prune and local neighborhood search, Eur. J. Oper. Res. 217 (2012) 609-618.

[22] E. K. Lee, T. Fox, I. Crocker, Simultaneous beam geometry and intensity map optimization in intensity-modulated radiation therapy, Int. J. Radiat. Oncol. Biol. Phys. 64 (2006) 301-320.

[23] E. Schreibmann, M. Lahanas, L. Xing, D. Baltas, Multiobjective evolutionary optimization of the number of beams, their orientations and weights for intensity-modulated radiation therapy, Phys. Med. Biol. 49 (2004) 747-770.

[24] D. M. Aleman, A. Kumar, R. K. Ahuja, H. E. Romeijn, J. F. Dempsey, Neighborhood search approaches to beam orientation optimization in intensity modulated radiation therapy treatment planning, J. Global Optim. 42 (2008) 587-607.

[25] G. J. Lim, J. Choi, R. Mohan, Iterative solution methods for beam angle and fluence map optimization in intensity modulated radiation therapy planning, OR Spectrum 30 (2008) 289-309.

[26] J. Stein, R. Mohan, X. H. Wang, T. Bortfeld, Q. Wu, K. Preiser, C. C. Ling, W. Schlegel, Number and orientation of beams in intensity-modulated radiation treatments, Med. Phys. 24 (1997) 149-160.

[27] H. E. Romeijn, R. K. Ahuja, J. F. Dempsey, A. Kumar, J. Li, A novel linear programming approach to fluence map optimization for intensity modulated radiation therapy treatment planing, Phys. Med. Biol. 48 (2003) 3521-3542.

[28] E. K. Lee, T. Fox, I. Crocker, Integer programming applied to intensitymodulated radiation therapy treatment planning, Ann. Oper. Res. 119 (2003) $165-181$.

[29] K. Cheong, T. Suh, H. Romeijn, J. Li, J. Dempsey, Fast Nonlinear Optimization with Simple Bounds for IMRT Planning, Med. Phys. 32 (2005) $1975-1975$.

[30] D. Craft, T. Halabi, H. Shih, T. Bortfeld, Approximating convex Pareto surfaces in multiobjective radiotherapy planning, Med. Phys. 33 (2006) 33993407 .

[31] G. J. Lim, M. C. Ferris, S. J. Wright, D. M. Shepard, M. A. Earl, An optimization framework for conformal radiation treatment planning, INFORMS J. Comput. 19 (2007) 366-380. 
[32] A. R. Conn, K. Scheinberg, L. N. Vicente, Introduction to Derivative-Free Optimization, SIAM, Philadelphia, 2009.

[33] C. Audet, J. E. Dennis Jr., Analysis of generalized pattern search methods, SIAM J. Optim. 13 (2003) 889-903.

[34] P. Alberto, F. Nogueira, H. Rocha, L. N. Vicente, Pattern search methods for user-provided points: Application to molecular geometry problems, SIAM J. Optim. 14 (2004) 1216-1236.

[35] C. Davis, Theory of positive linear dependence, Am. J. Math. 76 (1954) $733-746$.

[36] A. L. Custódio, H. Rocha, L. N. Vicente, Incorporating minimum Frobenius norm models in direct search, Comput. Optim. Appl. 46 (2010) 265-278.

[37] A. L. Custódio, L. N. Vicente, Using sampling and simplex derivatives in pattern search methods, SIAM J. Optim. 18 (2007) 537-555.

[38] MATLAB, The MathWorks Inc., http://www.mathworks.com.

[39] J. O. Deasy, A. I. Blanco, V. H. Clark, CERR: A Computational Environment for Radiotherapy Research, Med. Phys. 30 (2003) 979-985.

[40] J. O. Deasy, E. K. Lee, T. Bortfeld, M. Langer, K. Zakarian, J. Alaly, Y. Zhang, H. Liu, R. Mohan, R. Ahuja, A. Pollack, J. Purdy, R. Rardin, A collaboratory for radiation theraphy planning optimization research, Ann. Oper. Res. 148 (2006) 55-63.

[41] A. I. F. Vaz, L. N. Vicente, A particle swarm pattern search method for bound constrained global optimization, J. Global Optim. 39 (2007) 197-219. 